\title{
Le « livre du peuple ». La relation éditeur-public dans la presse francophone d'Amérique du Nord durant la Rébellion de 1837-1838
}

\author{
Julien Mauduit
}

Volume 72, numéro 4, printemps 2019

URI : https://id.erudit.org/iderudit/1065043ar

DOI : https://doi.org/10.7202/1065043ar

Aller au sommaire du numéro

Éditeur(s)

Institut d'histoire de l’Amérique française

ISSN

0035-2357 (imprimé)

1492-1383 (numérique)

Découvrir la revue

Citer cet article

Mauduit, J. (2019). Le « livre du peuple ». La relation éditeur-public dans la presse francophone d'Amérique du Nord durant la Rébellion de 1837-1838. Revue d'histoire de l'Amérique française, 72(4), 5-28.

https://doi.org/10.7202/1065043ar

\section{Résumé de l'article}

L'article étudie le pouvoir politique du public à travers son accès au journal, donc sa participation au débat public. En analysant les journaux francophones d'Amérique du Nord, il pose également un nouveau regard sur la Rébellion. Cette histoire des idées empirique soutient que le public francophone d'Amérique possédait une autonomie intellectuelle ainsi qu'un pouvoir d'intervention dans le débat public. Il démontre aussi que les journaux pro-révolutionnaires avaient une conception davantage démocratique des lecteurs. L'article insiste enfin sur l'importance d'étudier la circulation des idées dans l'opinion publique plutôt que la seule descente de celles-ci des éditeurs vers les lecteurs. 


\section{Le “livre du peuple». La relation éditeur-public dans la presse francophone d'Amérique du Nord durant la Rébellion de I837-I838}

Julien Mauduit

Université McMaster

RÉsumÉ • L'article étudie le pouvoir politique du public à travers son accès au journal, donc sa participation au débat public. En analysant les journaux francophones d'Amérique du Nord, il pose également un nouveau regard sur la Rébellion. Cette histoire des idées empirique soutient que le public francophone d'Amérique possédait une autonomie intellectuelle ainsi qu'un pouvoir d'intervention dans le débat public. II démontre aussi que les journaux pro-révolutionnaires avaient une conception davantage démocratique des lecteurs. L'article insiste enfin sur l'importance d'étudier la circulation des idées dans l'opinion publique plutôt que la seule descente de celles-ci des éditeurs vers les lecteurs.

ABSTRACT - This article analyses the public's political power through its access to newspapers, and therefore its participation in the public debate. By analyzing French North American newspapers, it sheds additional light on the Canadian Rebellion. As an empirical history of ideas, it argues that the public had an intellectual agency, and shows how the prorevolutionary newspapers shared more a democratic conception of its readers. The article also insists on the importance of considering the ideas' circulations rather than the elitist view of a top-down transmissions from the editors to the readers.

$\mathbf{L}$ e 15 novembre 1837, L'Abeille de la Nouvelle-Orléans publie un extrait du Libéral de Québec. Il ne s'agit pas d'un éditorial enflammé du patriote Robert-Shore-Milnes Bouchette, mais d'une correspondance du "Vieux de la Montagne», un Français exilé au Saguenay qui opine régulièrement dans les journaux. L'itinéraire de cette lettre offre un aperçu de l'espace public francophone d'Amérique du Nord, ce que Guillaume 
Pinson nomme la francosphère, un réseau polycentré, dynamique et cosmopolite reliant les communautés francophones du continent à la France et à l'Europe ${ }^{1}$. Par l'entremise des agents de journaux, des réseaux commerciaux ou des polémiques éditoriales, la presse francophone soutient la formation d'un espace d'échanges et de discussions publiques. Depuis Québec, Le Canadien d'Étienne Parent débat avec le Courrier des États-Unis de New York et L'Écho de la Nouvelle-Orléans; Le Populaire de Montréal échange avec l'éditeur du Courrier des États-Unis; L'Estafette de New York est lu en Louisiane comme au Bas-Canada ${ }^{2}$. Avec la Rébellion de 1837-1838², ces échanges se renforcent, aussi bien avec les États-Unis qu'avec la France ${ }^{4}$, Stephen Smith définissant même un «espace public transfrontalier» débattant sur la Rébellion'.

L'opinion publique s'est constituée avec le livre mais a connu son essor avec la presse, pensée et présentée comme le support privilégié de la discussion collective ${ }^{6}$. Dans son sens habermassien, une opinion publique

1. Guillaume Pinson, La culture médiatique francophone en Europe et en Amérique du Nord. De 1760 à la veille de la Seconde Guerre mondiale (Québec, Les Presses de l’Université Laval, 2016), p. 6-9. L'Abeille, 15 novembre 1837 et 6 décembre 1838 .

2. Pour saisir cet espace public francophone nord-américain: Gazette de Bâton Rouge, 17 mars 1838; Le Populaire, 10 juillet 1837; Yvan Lamonde, «Le Bas-Canada et Le Courrier des États-Unis de New York (18281840)", Les Cahiers des Dix, 56 (2002), p. 221-224; G. Pinson, La culture médiatique..., notamment p. 55-59 et 143; Jean Hamelin et André Beaulieu, "Aperçu du journalisme québécois d'expression française », Recherches sociographiques, 7, 3 (1966), p. 312; John Hare et Jean-Pierre Wallot, «Les entreprises d'imprimerie», dans Patricia Lockhart Fleming, Gilles Gallichan et Yvan Lamonde, dir., Histoire du livre et de l'imprimé au Canada (Montréal, Presses de l’Université de Montréal, 2004), vol. 1, p. 78-82; Fiona A. Black, «L'importation et la disponibilité du livre», dans P. Lockhart Fleming et al., dir., Histoire du livre..., p. 121-132; Yvan Lamonde et Andrea Rotundo, «Les commerces du livre et la librairie», dans P. Lockhart Fleming et al., dir., Histoire du livre..., p. 142-143; Anthony Grolleau-Fricard, «Le Courrier des États-Unis entre France, États-Unis et Canada (1828-1851)", thèse de doctorat (histoire), Université Panthéon-Sorbonne, 2009.

3. Soucieux de retranscrire certaines dynamiques transfrontalières de l'histoire, j'aborde l'événement dans son ensemble et par conséquent, j’adopte le singulier - la Rébellion - pour nommer la crise révolutionnaire de la fin des années 1830. Sur cette terminologie, lire en particulier Allan Greer, «La Rébellion de 1837-1838: une reconsidération", traduction de Denyse Beaugrand-Champagne, Bulletin d'histoire politique, 7, 1 (1998), p. 29-40.

4. Aurélio Ayala et Françoise Le Jeune, Les rébellions canadiennes de 1837 et 1838 vues de Paris (Québec, Les Presses de 1'Université Laval, 2011); Y. Lamonde, «Le Bas-Canada et Le Courrier...»; Maxime Dagenais, " $[\mathrm{T}]$ Hose Who Had Money Were Opposed to Us, and Those Who Were Our Friends Were Not the Moneyed Class": Philadelphia and the 1837-1838 Canadian Rebellions", American Review of Canadian Studies, 48 (2017).

5. Stephen Smith, «Within Arm’s Reach: Political Violence, Voluntary Organizing and the Borderland Press during the Canadian Rebellion, 1834-1842», thèse de doctorat (histoire), Queen's University (2017). Smith se penche sur la presse anglophone et le Haut-Canada, mais des liens et des parallèles ressortent avec l'espace public francophone. Les patriotes étaient de grands lecteurs de la presse américaine : LouisGeorges Harvey, Le printemps de l'Amérique française. Américanité, anticolonialisme et républicanisme dans le discours politique québécois, 1805-1837 (Montréal, Boréal, 2005).

6. Pierre Karila-Cohen, «L'opinion», dans Pierre Karila-Cohen et al., dir., La civilisation du journal. Histoire culturelle et littéraire de la presse française au $19^{e}$ siècle (Paris, Nouveau Monde éditions, 2011), p. 1355 ; Géraldine Mulhmann, «La démocratie médiatique», dans le même ouvrage, p. 1375 ; Micheline Cambron et Hans- 
se crée lorsque le jugement critique quitte la sphère privée pour s'adresser à la société civile et influer sur la politique. C’est ce phénomène que Jeffrey McNairn a étudié pour le Haut-Canada de la première moitié du XIX siècle, soit le passage à un monde politique où l'opinion publique est capable non seulement de saisir les débats, mais surtout de contraindre les pouvoirs législatifs et exécutifs à composer avec le regard critique du public. Cette transformation est assimilée à la naissance d'une «deliberative democracy ${ }^{7}$. Gilles Gallichan et Maurice Lemire ont également souligné pour le Bas-Canada l'importance de la presse dans la formation d'une opinion publique, résultat de l'essor de ce que les historien(ne)s qualifient de presse d'opinion ${ }^{8}$. Pierre Rajotte estime pour sa part que les années 1830 voient les dynamiques de l'espace public évoluer profondément, car certaines élites font délibérément déborder la discussion hors des cercles bourgeois'. Si les acteurs de l'histoire et les universitaires s'accordent pour identifier l'existence d'une réflexion critique au sein du public, il est bien plus délicat d'en cerner ses mécanismes, son étendue dans le corps social (la délimitation sociologique du public), et encore plus la nature et le sens de cette "opinion».

La décennie 1830 a été le témoin d'une révolution médiatique pendant laquelle la presse devient plus accessible, démocratisant par ce biais la discussion et la réflexion ${ }^{10}$. Apparaissent, par exemple, les penny papers aux États-Unis et la presse ouvrière ou féminine en France ${ }^{11}$. L'éditeur est au

Jürgen Lüsenbrick, «Presse, littérature et espace public: de la lecture et du politique», Études françaises, 36, 3 (2000), p. 133-134.

7. Jeffrey McNairn, Capacity to Judge. Public Opinion and Deliberative Democracy in Upper Canada, 17911854 (Toronto, University of Toronto Press, 2000). Pour un regard plus critique sur le processus, lire Carol Wilton, Popular Politics and Political Culture in Upper Canada, 1800-1850 (Montréal et Kingston, McGillQueen's University Press, 2000).

8. Gilles Gallichan, Livre et politique au Bas-Canada (Québec, Septentrion, 1991); Maurice Lemire, dir., La vie littéraire au Québec. Vol. II 1806-1839 (Québec, Les Presses de l'Université Laval, 1992). Voir également: Nova Doyon, dir., 1811, de Québec à Montréal, essor de la presse et affirmation d'une parole publique francophone (Montréal, Petit musée de l'impression, 2009); M. Cambron et H.-J. Lüsenbrick, «Presse, littérature et espace public...", p. 133-134; J. Hamelin et A. Beaulieu, "Aperçu du journalisme... », p. 309-311; Claude Galarneau, "La presse périodique au Québec, de 1764 à 1859 », Mémoires et comptes rendus de la Société royale du Canada, 4, 12 (1984), p. 143-166; Mylène Bédard, Écrire en temps d’insurrections. Pratiques épistolaires et usages de la presse chez les femmes patriotes (1830-1840) (Montréal, Presses de l’Université de Montréal, 2016), p. 41-47.

9. Pierre Rajotte, «La sociabilité littéraire au Québec: de l’usage public de la raison à la reconnaissance d'une légitimité fondée sur un principe de compétence», Voix et images, 27, 2 (2002), p. 203-204.

10. Alain Vaillant et Marie-Ève Thérenty, 1836: l'an I de l'ère médiatique, étude littéraire et historique du journal La Presse, d'Émile de Girardin (Paris, Nouveau Monde éditions, 2001).

11. Richard D. Brown, Knowledge Is Power: The Diffusion of Information in Early America, 1700-1865 (New York, Oxford University Press, 1989), p. 272-296; Charles G. Steffen, «Newspapers for Free: The Economies of Newspaper Circulation in the Early Republic», Journal of the Early Republic, 23, 3 (2003), p. 282-284 ; James L. Crouthamel, «The Newspaper Revolution in New York 1830-1860 ", New York History, 45, 2 (1964), p. 91-113; P. Karila-Cohen, «L’opinion»..., p. 1363 ; Mélanie Guérimand, «Du Papillon pour 
cœur de cette révolution des médias, et le journal devient le nœud de transformations globales qui créent une véritable civilisation du journal ${ }^{12}$. Nous sommes alors, rappelons-le, en pleine ère des révolutions atlantiques, une vague qui s'étend aux colonies britanniques d'Amérique du Nord avec la Rébellion ${ }^{13}$. L'éditeur, souvent propriétaire, parfois imprimeur de profession, personnifie le journal - il est, individuellement, l'esprit de sa feuille, celui qui parle au public, et avec lequel le public et les correspondants échangent. Le journal est donc un espace polyphonique qui crée une interdiscursivité entre le monde politique, celui des lettres, et le public ${ }^{14}$. Il joue son double rôle d'être à l'origine de l'opinion du lecteur ainsi que son porte-voix. Parallèlement, la pratique de la lecture est elle-même publique et il n'est plus nécessaire d'être alphabétisé pour lire un journal ${ }^{15}$. La relation éditeur-public est donc essentielle pour évaluer la réalité du caractère démocratique du journal ${ }^{16}$.

Ce texte porte sur une réalité historique et empirique de l'opinion publique: l'interaction et l'échange entre l'éditeur et son public (sa figure, sa voix, son influence). Si la presse véhicule l'idée que le public est doué de réflexion critique, et que le journal est le vecteur qui permet l'existence de l'opinion publique, alors l'éditeur doit être le garant de l'échange critique $^{17}$. L'opinion du public est en effet régulièrement présentée par les éditeurs comme le tribunal de l'histoire, et le journal le «livre du peuple». À l'heure de la Rébellion, comment les éditeurs francophones du continent ont-ils œuvré pour faire de leur journal ce lieu de formation des opinions, l'opérateur dynamique qui fait circuler les idées et organise les échanges? Existe-t-il, à cet égard, une différence éditoriale selon les sensibilités politiques? L'espace francophone nord-américain de la fin des années 1830 permet une telle enquête puisque les journaux favorables à une révolution

les dames au Conseiller pour les femmes: les voix de femmes dans la presse féminine lyonnaise sous la Monarchie de Juillet», dans Elina Absalyamova et Valérie Stiénon, dir., Les voix du lecteur dans la presse française du XIX siècle (Limoges, PULIM, 2018), p. 73-83.

12. P. Karila-Cohen et al., dir. La civilisation du journal... Lire également Guillaume Pinson, L'imaginaire médiatique. Histoire et fiction du journal au XIXe siècle (Paris, Classiques Garnier, 2013).

13. Eric J. Hobsbawm, L'ère des révolutions: 1789-1848 (Bruxelles, Complexe, 2000 [1962]); Michel Ducharme, Le concept de liberté au Canada à l'époque des révolutions atlantiques, 1776-1838 (Montréal et Kingston, McGill-Queen's University Press, 2010).

14. E. Absalyamova et V. Stiénon, dir., Les voix du lecteur...

15. Pour le cas du Bas-Canada: Allan Greer, Habitants et Patriotes. La Rébellion de 1837 dans les campagnes du Bas-Canada (Montréal, Boréal, 1997 [1993]), p. 131; Louis-Georges Harvey, «La lecture publique au Québec de 1850 à 1900: vers la démocratisation du livre », Documentation et bibliothèques, 41, 3 (1995), p. 143-146.

16. E. Absalyamova et V. Stiénon, dir., Les voix du lecteur..., p. 27.

17. P. Karila-Cohen, «L'opinion»..., p. 1358-1360. 
au Bas-Canada, comme La Minerve de Montréal, Le Libéral de Québec ou L'Estafette de New York s'opposent frontalement à une presse francophone que nous pouvons qualifier de constitutionnaliste ou loyaliste qui soutient les autorités britanniques contre la fièvre républicaine: Le Courrier des États-Unis de New York, L'Ami de l'ordre et des lois et Le Populaire de Montréal, et Le Canadien de Québec.

Les historien(ne)s du Bas-Canada insistent tout particulièrement sur le lien entre la formation de l'opinion publique et l'affirmation d'une conscience nationale canadienne-française ${ }^{18}$. Mais cette presse a aussi encouragé la contestation politique, donc l'opinion à opiner, le public à s'immiscer dans le débat politique. Ce phénomène a généré une vitalité de la discussion et a alimenté les accords aussi bien que les désaccords, créant un véritable champ médiatique ${ }^{19}$. Au moment de la Rébellion, le ton s'est durci et les oppositions se sont raffermies. À l'inverse d'une historiographie qui tend à insister sur les oppositions nationales du milieu journalistique, nous aborderons donc, pour mieux saisir les réalités de «l'opinion» de la francosphère, les oppositions qui traversent la presse francophone, notamment sur ses divisions politiques, qui ne sont que très partiellement rapportées dans la littérature savante ${ }^{20}$.

Ce texte combine donc histoire de la presse, histoire politique et histoire des idées pour apporter un nouveau regard sur la Rébellion et pour mieux matérialiser certains mécanismes de l’opinion publique. Malgré un discours de principes parfois similaire, les démarches des éditeurs sont radicalement différentes dans leur rapport matériel et figuré au public. Le corpus a été constitué afin de retranscrire ces différences, réduire l'attraction exercée par certains titres comme La Minerve et Le Courrier des ÉtatsUnis, mais également pour comparer et connecter les titres de la francosphère nord-américaine. Fidèle aux idéaux républicains, la presse pro-patriotes considère davantage le public dans ses colonnes et ses

18. M. Lemire, dir., La vie littéraire...; Micheline Cambron, dir., Le Journal Le Canadien. Littérature, espace public et utopie (1836-1845) (Montréal, Fides, 1999); Bernard Andrès, La conquête des lettres au Québec (1759-1799). Anthologie (Québec, Les Presses de l’Université Laval, 2007); N. Doyon, dir., 1811...; G. Pinson, La culture médiatique..., p. 78-79; Denis Monière, «Ludger Duvernay, le journal La Minerve et la construction identitaire canadienne», dans N. Doyon, dir., 1811..., p. 59-83; Nova Doyon, Formation des cultures nationales dans les Amériques. Le rôle de la presse dans la constitution du littéraire au Bas-Canada et au Brésil au début du XIXe siècle (Québec, Les Presses de l’Université Laval, 2012), p. 57.

19. G. Pinson, La culture médiatique..., p. 80.

20. Il faut par conséquent nuancer la centralité de l'opposition entre publications francophones et anglophones qui a été suggérée par J. Hamelin et A. Beaulieu, «Aperçu du journalisme... », p. 309-310. Par exemple, Gilles Laporte n'insiste pas sur l'opposition entre Étienne Parent et les républicains: Brève histoire des patriotes (Québec, Septentrion, 2015), p. 63, au contraire d’Yvan Lamonde, Histoire sociale des idées au Québec, 1760-1896 (Montréal, Fides, 2000), p. 238. 
réflexions. Mais Le Populaire, loyaliste convaincu, énonce également des principes démocratiques, et participe au renouvellement de la presse en invitant les femmes à s'exprimer. En outre, l'ancien porte-voix de la contestation bas-canadienne, Étienne Parent, fait preuve d'une surprenante condescendance envers le public. Au-delà de ces différentes démarches qui sont loin d'être déterminées uniquement par l'orientation politique, adopter un regard transfrontalier permet surtout de constater que la remise en cause de l'ordre établi par certains a encouragé une réflexion publique générale.

\section{LA PRESSE FRANCOPHONE ET LA RÉBELLION}

Il est délicat d'isoler les Canadas des États-Unis pour analyser la Rébellion, surtout à partir de novembre $1837^{21}$. En nous limitant à la presse du BasCanada, nous pourrions par exemple en conclure que la parole révolutionnaire s'est tue avec le début du soulèvement, ce qui n'est pas le cas du fait, précisément, de la présence de journaux étasuniens. Alors que les feuilles radicales ont dû fermer dès les premiers jours du conflit militaire, le 4 mai 1838 le Conseil spécial rend une ordonnance obligeant les journaux du BasCanada à obtenir une autorisation administrative avant publication ${ }^{22}$. Pour lire une critique de la politique britannique, le lecteur doit se procurer des journaux étasuniens. Fin 1838, les autorités emprisonnent même Étienne Parent qui avait pourtant clairement affiché sa loyautée ${ }^{23}$. Les répercussions de cette censure sont continentales, l'actualité canadienne étant principalement relatée à partir des plumes loyalistes ${ }^{24}$. La francosphère du continent a notamment œuvré pour faire contrepoids face à la censure.

Pour reprendre Malesherbes qui observe que «les gens de lettres sont au milieu du public dispersé ce qu'étaient les orateurs de Rome et d'Athènes au milieu du public assemblé25 ", les journaux sont dans les années 1830 au centre du public francophone dispersé en Amérique. Nous

21. Julien Mauduit, " "Vrais républicains” d'Amérique : les patriotes canadiens en exil aux États-Unis, 1837-1842", thèse de doctorat (histoire), UQAM, 2016.

22. Maxime Dagenais, " "La mort du conseil spécial est la meilleure action que le corps ait faite durant sa vie $[\ldots]$ ]": The French-Canadian Press and the Special Council of Lower Canada, 1838-1841 ", Quebec Studies, 62 (2016), p. 26-27. Les journaux étasuniens pro-patriotes étaient saisis dès le mois de mars: L'Estafette des 6, 8 et 17 avril 1838; Amédée Papineau, Journal d'un Fils de la Liberté, 1838-1855 (Québec, Septentrion, 2010), p. 161. Bouchette accuse le Bureau des Postes de filtrer la circulation des journaux dès l'automne 1837: Le Libéral, 8 septembre 1837.

23. M. Dagenais, “"La mort du conseil spécial...”", p. 36-37.

24. Lire par exemple les plaintes de L'Abeille, 30 novembre 1837.

25. Cité par Franck Salaün, "Les livres nécessaires et l'opinion publique selon Malesherbes», dans Bertrand Binoche et Alain-Jacques Lemaître, dir., L'opinion publique dans l'Europe des Lumières : stratégies et concepts (Paris, Armand Colin, 2013), p. 49. 
pouvons même parler d'un essor du lectorat français aux États-Unis ${ }^{26}$. À la fin des années 1830, le Courrier des États-Unis de New York compte 2200 abonnés et L'Abeille de la Nouvelle-Orléans 2500, quand le plus grand journal étasunien imprime 11500 exemplaires par édition ${ }^{27}$. Au début de 1838, Frédéric Gaillardet, homme de lettres français voyageant en Amérique du Nord, envisageait d'établir le premier journal qui s'adresserait à ces dizaines de milliers de «Français» d'Amérique. Depuis New York, "point central [...] du triangle franco-américain», il «ne chercherait point à empêcher que le Canadien se soumit au joug anglais, et le Louisianais au joug américain ", mais à rassembler ce public autour d'un journal qui éviterait les clivantes questions politiques ${ }^{28}$.

Dans les années 1830, les éditeurs francophones bas-canadiens déclarent tous défendre les Canadiens français, ce que remarque Gaillardet: «Notre langage, nos usages et nos mours, telle est la devise inscrite en tête de presque tous les journaux français du Canada, et de ceux mêmes qui sont les plus soumis au gouvernement anglais ${ }^{29}$.» Cependant, ils sont loin d'être unanimes sur la marche politique à adopter. L'attachement commun à la culture française ne détermine donc pas la teneur politique de ces journaux. Du fait de son soutien aux constitutionnalistes, Étienne Parent, célèbre éditeur du Canadien, est ainsi associé par la presse républicaine aux apostats du Populaire et de l'Ami du Peuple ${ }^{30}$. Parent partage ce constat. Pour l'éditeur-propriétaire du Canadien, il y a «six journaux publiés en français dans ce pays, et appartenant à diverses nuances politiques; [...] deux soutiennent une politique contre laquelle s'élèvent les quatre autres journaux, chacun selon ses vues particulières ${ }^{31} »$. Il trace la ligne de fracture entre l'esprit révolutionnaire de La Minerve et du Libéral, et les quatre journaux loyaux dont il fait partie. Parent se fait pourtant régulièrement accuser de soutenir Louis-Joseph Papineau. Il s'en défend en répétant qu'il

26. Marieke Polfliet, «Émigration et politisation. Les Français de New York et La Nouvelle-Orléans dans la première moitié du XIX ${ }^{\mathrm{e}}$ siècle (1803-1860)", thèse de doctorat (histoire), Université de Nice-Sophia Antipolis, 2013, p. 360.

27. Pour le Courrier et l'Abeille, M. Polfliet, "Émigration et politisation...», p. 364-365; J. L. Crouthamel, "The Newspaper Revolution... », p. 96.

28. Frédéric Gaillardet au ministre des Affaires étrangères, 18 mars 1838, correspondance retranscrite par M. Polfliet, «Émigration et politisation... », p. 883-884.

29. Retranscrit par M. Polfliet, "Émigration et politisation...» p. 883. Voir également Christiane Campagna, «Le rôle de la presse selon les propriétaires et rédacteurs des journaux montréalais, 18301880 ", mémoire de maîtrise (histoire), UQAM, 1998, p. 75.

30. Pour saisir la vive polémique, lire en particulier Le Libéral des 5 juillet, 25 août et 22 septembre 1837, et Le Canadien des 19 et 23 juin 1837. Sur sa prise de position favorable aux constitutionnalistes, Le Canadien, 30 juin 1837.

31. Le Canadien, 9 octobre 1837. 
s'oppose à l'interférence des Étasuniens, à la déstabilisation de l'ordre social et aux dangers d'une "déclaration de guerre anticipée», prônés par les patriotes ${ }^{32}$. Il reproduit même des éditoriaux appelant à la répression des agitateurs ${ }^{33}$. Alors que la tension polarise l'espace public, Parent se retrouve finalement dans "une espèce de juste-milieu », isolé politiquement, car il reconnaît aussi une certaine légitimité à la contestation à laquelle il a participé dans le passé ${ }^{34}$.

Au Bas-Canada comme à New York, l'échiquier de la presse francophone se restructure sur des lignes politiques en 1837. À Québec, pour tenter d'occuper l'espace laissé vacant par Parent, le journal bilingue Le Libéral est lancé en juin pour s'adresser aux patriotes de la ville ${ }^{35}$. La feuille soutient Papineau, défend la légitimité de la contestation et glorifie la perspective d'une révolution. Dans une réflexion presque binaire, l'éditeur-propriétaire R.-S.-M. Bouchette refuse de considérer les subtilités du loyalisme de Parent, car il n'existe à ses yeux que deux positions: la soumission ou la résistance ${ }^{36}$. Il l'accuse de "corrompre l'opinion» et de «flétrir aux yeux de l'univers [...] l'honneur et le caractère du peuple Canadien ${ }^{37}$. Bouchette critique notamment le nationalisme strictement franco-canadien de Parent et insiste sur la définition du peuple du Parti patriote: dépasser les «malheureuses distinctions de personnes déduites du seul accident de l'origine», afin d'accueillir "tous les hommes comme compatriotes $»^{38}$.

Si la loyauté envers l'Empire britannique est une boussole politique déterminante, la discussion publique est loin d'être binaire. Comme le fait remarquer Parent, les éditeurs ont chacun des «vues particulières». L'émigré français Leblanc de Marconnay, directeur du Populaire de Montréal lancé en avril 1837, est un autre éditeur avec qui Bouchette croise régulièrement le fer. Marconnay est lui aussi accusé de trahison, car il avait collaboré à $L a$ Minerve $^{39}$, tout comme le propriétaire du journal et cousin de Papineau, 1837.

33. Le Canadien, 12 juillet 1837.

34. Sur le «juste milieu » : Le Canadien, 19 juin 1837 et Le Populaire, 31 juillet 1837. Pour lire une analyse faisant de Parent un éditeur d'une grande influence: J. Hamelin et A. Beaulieu, «Aperçu du journalisme... », p. 310-314. Voir aussi G. Laporte, Brève histoire..., p. 152 et 157.

35. Pour Gérard Laurence («Les journaux...», p. 250), les journaux bilingues "disparaissent pratiquement» après 1820. Gilles Gallichan, «La censure politique», dans P. Lockhart Fleming et al., dir., Histoire du livre..., p. 347.

36. Le Libéral, 22 septembre 1837.

37. Le Libéral, 17 et 28 juin, et 22 septembre 1837.

38. Le Libéral, 17 juin 1837, prospectus.

39. Le Libéral, 17 juin 1837. 
Léon Gosselin ${ }^{40}$. Le Populaire prêche ouvertement la loyauté envers les autorités. Il met en garde contre l'ingérence des voisins du Sud et soutient le besoin d'une répression sévère, opinion que l'éditeur du Canadien n'exprime que du bout des lèvres. Ce qui les effraie est la «torche incendiaire $[\ldots]$ capable d'embraser l'édifice social et d'en détruire la solidité» que contribuent à allumer les éditeurs patriotes ${ }^{41}$.

Nous retrouvons ces mêmes divisions aux États-Unis ${ }^{42}$. Ni l'ascendance française ni l'appartenance à une république ne déterminent une convergence d'opinion. L'Abeille de la Nouvelle-Orléans sympathise avec les patriotes, notamment parce que les Louisianais sont esclavagistes et de ce fait hostiles aux Britanniques. Pour Jérôme Bayon, éditeur et propriétaire du journal, les patriotes bas-canadiens ne forment pas « une populace en démence, mais un peuple réfléchi, courageux, puissant de force et d'énergie». Son soutien ne souffre d'aucune nuance dans les premières semaines du conflit, mais la neutralité officielle du président Martin Van Buren le convainc d'adopter un ton plus mesuré: il existe des «circonstances où l'opinion des gouvernés se trouve en opposition avec la sagesse des gouvernants ${ }^{43}{ }^{\prime}$. Le Courrier des États-Unis de New York est au contraire un adversaire avoué: "Nos couleurs nationales serviront donc de ralliement à un tas d'ignorants qui ne savent ni lire ni écrire ${ }^{44}$. "Selon Gaillardet, le Courrier a eu le tort "d'avoir pris un parti cruellement hostile aux Canadiens insurgés», ce qui a "porté un coup funeste à ce journal ${ }^{45}$ ». Son concurrent new-yorkais rappellera quelques mois plus tard qu'il «s'était aliéné tous ses lecteurs " par son verbe antipatriotique ${ }^{46}$. Comme à Québec, la position politique du Courrier encourage la fondation d'un concurrent, L'Estafette ${ }^{47}$. Lancé durant les premières semaines de la Rébellion par un immigré anglais, H. D. Robinson, il devient le principal

40. Gérard Laurence, «Léon Gosselin», Dictionnaire Biographique du Canada, <www.biographi.ca/ fr/bio/gosselin_leon_7F.html>. Les Patriotes, comme certains historiens (J. Hamelin et A. Beaulieu, "Aperçu du journalisme...», p. 310), affirment que Debartzch est lié au journal, ce dont se défend Marconnay: Le Populaire, 21 juin 1837.

41. Le Populaire, 10 avril 1837. Lire «De la nécessité d’employer la fermeté» du 23 juin 1837 pour saisir l'aversion du journal envers le Parti patriote.

42. Pour un tableau politique de cette presse, sans lien direct avec la Rébellion: M. Polfliet, "Émigration et politisation...», p. 397-403.

43. Sur le soutien sans failles: L'Abeille, 18 novembre et 7 décembre 1837 . Pour lire le ton plus modéré: L'Abeille, 16 janvier 1838.

44. Le Courrier des États-Unis, 29 novembre 1837; La Minerve, 9 octobre 1837; Y. Lamonde, «Le Bas-Canada...»".

45. Gaillardet cité par M. Polfliet, «Émigration et politisation... », p. 883-844.

46. L'Estafette, 3 juillet 1838. Voir aussi le numéro du 3 avril 1838.

47. L'Estafette, 24 avril 1838. 
avocat des républicains canadiens dans la presse francophone d'Amérique du Nord. Chaque exemplaire consacre de longues colonnes aux événements, défendant sans concessions «l'entrée dans la carrière des révolutions " des Canadas, un peuple qui possède un droit naturel de «déchirer un contrat qu'il n'a jamais ratifié $»^{48}$. L'Estafette devient un rouage majeur des réseaux révolutionnaires: il ouvre ses colonnes à Ludger Duvernay, aide William Lyon MacKenzie à implanter son journal et se fait globalement leur porte-voix ${ }^{49}$. En observant la répression, et probablement en constatant son impopularité, le Courrier évolue en 1838 vers une position plus favorable aux patriotes ${ }^{50}$. La volte-face est spectaculaire, comme le sont, à un degré moindre, celles du Canadien et du Populaire ${ }^{51}$.

Les prises de position ne sont pas binaires et elles évoluent durant les mois de crise, ce qui témoigne de la vitalité des opinions. La discussion déborde des contextes locaux. Dès le début du conflit militaire, la discussion s'engage entre les éditeurs loyalistes du Bas-Canada et les journaux étasuniens pro-patriotes qui circulent dans la colonie, prenant par ce biais une place déterminante dans les événements en tant que principales feuilles favorables à la révolte. L'Estafette, en particulier, s'indigne des justifications de L'Ami du Peuple des massacres commis par les troupes de Sa Majesté. Pour Robinson, le journal montréalais «crache sur les cadavres »; "Honte à jamais à ces apostats du journalisme qui, par intérêt ou par frayeur, s'agenouillent devant le pouvoir $\aleph^{52}$. La prompte réponse - «nous avons fait notre devoir ${ }^{53}$ » - met en marche un échange acide qui, finalement, mobilise les principes politiques et la logique davantage que l'insulte ou l'évidente mauvaise foi. Les deux éditeurs dissertent, à travers les frontières, sur le patriotisme, le droit des peuples à modifier les lois, la légitimité de la violence d'État et la loi martiale, sujets nécessitant de longs argumentaires $^{54}$. En comparaison aux controverses bas-canadiennes, il est même surprenant de constater la place qu'offre Robinson dans son journal aux propos de son concurrent. Volontairement ou non, cette pratique s'adresse manifestement à l'esprit critique du public.

48. Citations tirées de L'Estafette, du 5 janvier et du 20 février 1838.

49. Fonds Ludger Duvernay, Jean Thompson à Duvernay, 6 novembre 1838, The Canadian and Numismatic Journal, VIII (1910), p. 76-77. L'Estafette des 17 avril 1838 (Duvernay) et 3 avril 1838 (MacKenzie).

50. Y. Lamonde, «Le Bas-Canada...», p. 226-233. Lire par exemple Le Courrier des États-Unis des 31 octobre et 10 novembre 1838 .

51. Y. Lamonde, «Le Bas-Canada...»; M. Dagenais, «"La mort du conseil spécial...”».

52. L'Estafette, 12 janvier, 20 et 23 février 1838.

53. L'Ami du peuple de l'ordre et des lois, 20 janvier 1838.

54. L'Estafette des 27 février et 2 mars 1838. 


\section{L’ÉDITEUR ET SON PUBLIC}

La Révolution française a fait naître le mythe du journal comme support de l'échange permanent entre citoyens, l'une des conditions du progrès démocratique. Dans les années 1830, le public s'élargit et l'éditeur se réinvente dans cette ère médiatique nouvelle ${ }^{55}$. Au Bas-Canada, le peuple devient un mot omniprésent ${ }^{56}$. L'éditeur d'une presse d'opinion est un homme public, politiquement engagé et au centre de la discussion. Ses éditoriaux constituent sa tribune en même temps que le cœur de son journal $^{57}$. Chez les éditeurs francophones d'Amérique du Nord, plusieurs principes font consensus ${ }^{58}$. Ils déclarent notamment que le journal est un vecteur d'émancipation. Pour Parent, la presse est «la seule bibliothèque du peuple»; "le savoir est une puissance et chaque nouveau lecteur ajoute à la force populaire $»^{59}$. Bouchette précise que les «journaux publics sont les seules sources où je puise mes connaissances ${ }^{60} »$. Ainsi, le journal est présenté comme «le livre du peuple», la pierre angulaire de la transmission d'un savoir libérateur. Les termes utilisés pour définir leurs lignes politiques sont relativement similaires. Bien que prônant la répression contre le Parti patriote, Marconnay se présente comme "patriote» et insiste sur son esprit «libéral». Le Parti patriote et sa presse n'ont pas le monopole de l'idéal démocratique puisque l'éditeur loyaliste annonce «qu'aucune classe de la société ne sera exclue des intérêts dont LE POPULAIRE devient l'ardent avocat $»^{61}$. Ces convergences démontrent que nous ne pouvons pas nous contenter de constater, superficiellement, telle ou telle occurrence dans leurs écrits si nous voulons comprendre leurs discours.

Leur conception du public, la figure du lecteur et la relation que les éditeurs entretiennent avec lui démontrent toutefois de significatives divergences. Parent défend l'idée que le journal doit être une «boussole de l'opinion publique» écrit par des hommes «chargés de conduire nos destinées ${ }^{62}$. L'éditeur doit orienter la réflexion. Parent ne prétend pas

55. Corinne Saminadar-Perrin, "Avatars journalistiques de l'éloquence publique», dans P. KarilaCohen et al., La civilisation du journal..., p. 669-671.

56. G. Pinson, La culture médiatique..., p. 80.

57. G. Laurence, «Les journaux...», p. 251.

58. Aspect de l'histoire de la presse qui a été souligné par C. Campagna, «Le rôle de la presse... ».

59. Ces mots proviennent du numéro de relance du Canadien, 7 mai 1831, partiellement cité par M. Bédard, Écrire en temps d'insurrections..., p. 42.

60. Le Libéral, 17 juin 1837.

61. Le Populaire, 10 avril et 21 juin 1837. Majuscules dans l'original.

62. Le Canadien, 21 juin 1837. 
retranscrire l'opinion publique, mais la guider. Il ne réfère pas non plus à l'opinion publique comme force politique autonome. Son argumentaire s'appuie parfois sur cette opinion, mais uniquement lorsqu'elle concorde avec sa propre réflexion, pour prouver la justesse de ses propos. C'est le cas quand il traite des résolutions de l'assemblée publique de Berthier: il affirme avoir eu raison de dénoncer la contrebande et l'implication des Américains, car ces vues ont été ignorées lors de cette assemblée. La position des habitants est le "point juste» du discours de raison, celui «où l'opinion publique devait s'arrêter ${ }^{\prime 63}$. Cette démarche intellectuelle infantilise le public puisque la hiérarchie est affirmée ; elle est également paradoxale, car c'est la présence de son opinion au sein de la population qui lui confère sa véracité. Son ton est parfois hautain, comme lorsqu’il déplore «que de petits et minces journaux ${ }^{64}$ comme le Libéral, publiés à un prix qui les met à la portée des classes ouvrières, fassent beaucoup de mal parmi des gens sans instruction et sans expérience ${ }^{65} \%$. Ce n'est pas le fait que des gens sans instruction lisent un journal qui pose problème, mais l'accès de ce public au message du Libéral, car Parent n'a pas confiance en leur capacité à développer une critique du journal ${ }^{66}$. La conception de la relation éditeur-public de Parent est fondée sur la transmission de la vérité par l'homme de lettres, à des fins politiques précises, une démarche qui réduit l'espace de la discussion.

Le Libéral utilise régulièrement les correspondances du public pour s'opposer à Parent. Un «Anti-Coercitionniste» ironise ainsi sur la position de l'ancien éditeur patriote: "Oui, oui, que l'opinion publique se taise à la vue des mesures iniques et atroces du ministère britannique ${ }^{67}$. » Le député Augustin-Norbert Morin est alors convaincu que Parent ne triomphera pas, car il y a «trop d'esprit public» parmi la population: «les connaissances sur nos affaires publiques sont assez répandues $»^{68}$. Le public éclairé de Bouchette et Morin est doué d'un «esprit», davantage que d'une simple opinion, ce qui le conduit naturellement vers la contestation.

Marconnay intègre plus explicitement le public que ne le fait Parent au sein d'une conception élargie. Il prend en particulier grand soin d'inviter les femmes à s'exprimer, principalement pour leurs talents littéraires, car «rien ne justifie» l’idée d’une supériorité masculine. Marconnay critique

63. Le Canadien, 26 juin 1837.

64. Il s'agit d'une expression régulièrement utilisée pour dénigrer son rival.

65. Le Canadien, 23 octobre 1837.

66. Le Canadien 7 juillet 1837.

67. Le Libéral, 5 juillet 1837.

68. Correspondance de Morin dans Le Libéral, 26 juillet 1837. 
ses concurrents qui ne font que prétendre faire parler le peuple, alors qu’en réalité ils "proscrivent la liberté de raisonnement». Pour y remédier, il annonce que son devoir est de ne "jamais proscrire une réponse» et de publier les propos de ses adversaires afin de laisser le public forger son opinion. Par conséquent, il affirme qu'il sera impossible de l'«accuser de chercher à abuser ce maître commun, LE PEUPLE». Cependant, il voit aussi la presse comme «la véritable Ecole Normale où tous les citoyens, de quel que rang qu'ils soient, viennent puiser la science comme la conscience de leurs devoirs politiques et privés». Il présente même Le Populaire comme le vecteur qui propagera «une saine éducation morale et politique ${ }^{69}$. Si le discours témoigne d'une certaine démocratisation, est-il possible d'accorder la volonté de créer un espace de discussion avec celle de propager une "saine éducation", à moins bien sûr de prédéfinir la nature des échanges?

La conception du public par H. D. Robinson est là aussi singulière. Il est l'éditeur qui s'y réfère le moins, en termes quantitatif, et il ne fait pas de discours de principes sur le sujet. Il ne cherche pas à multiplier les correspondances de lecteurs dans ses colonnes. Il refuse par exemple de publier une correspondance signée «Vox Populi», pourtant en accord avec sa ligne éditoriale, car justement elle n'apporte rien: "nous pensons que l'article précédent nous dispense de l'insérer, car il exprime des sentiments qui sont ceux de notre correspondance ${ }^{70} »$. La voix du lecteur est donc ici maintenue hors du journal au nom de la fluidité du numéro. Autre exemple, Robinson ne publie pas une correspondance favorable à la révolution canadienne qui accuse l'armée britannique d'avoir illégalement envahi le territoire des États-Unis pour attaquer les révolutionnaires, ce qu'il se refuse de croire pendant quelques jours. Il précise aussi que les correspondances subiront sa relecture: «nous prierons ceux qui voudront bien nous adresser des communications de se faire connaitre, certains qu'ils peuvent être de notre discrétion: il nous sera possible alors de nous entendre avec eux pour les changements qui nous paraitraient convenables $^{71}$.» Ce qui est convenable ne réfère pas à une opinion politique, mais au sérieux de la réflexion. Cette pratique éditoriale ne signifie pas un mépris pour les lecteurs qui souhaitent opiner. Sa représentation du public n'apparaît qu'en creux. En publiant le texte d'un membre de l'Académie

69. Le Populaire, 10 avril 1837. Majuscules dans l'original.

70. L'Estafette, 5 janvier 1838.

71. L'Estafette, 9 janvier 1838 . Voir aussi L'Estafette du 19 janvier 1838 sur la responsabilité de l'armée britannique. 
française, Jean-Pons-Guillaume Viennet, "Philosophie de l'opinion publique», Robinson souligne qu'il regrette que la presse y soit maltraitée, et que l'auteur y peigne une sombre image de la capacité intellectuelle du public $^{72}$. La publication de ce long texte illustre la place que Robinson accorde aux opinions contraires aux siennes, ce qui doit permettre à ses lecteurs de mieux juger. Il reste finalement fidèle à son idée initiale d'affirmer son opinion sans condamner la discussion: "nous voulons trop que l'on respecte nos opinions, pour ne pas respecter celles des autres ${ }^{73} »$. Bien qu'il estime clairement s'adresser à un lectorat doué de raison, la pratique éditoriale de Robinson maintient aussi une stricte hiérarchie dans les échanges publics, au profit de l'homme de lettres.

R. S. M. Bouchette défend également sa propre relation éditeur-lecteur. Il martèle que l'opinion publique doit orienter la politique. "C'est cette OPINION PUBLIQUE si puissante dans le destin des Peuples» que Le Libéral annonce vouloir servir. Le journal serait né d'une "conviction intime et inébranlable que les intérêts les plus chers du Pays, la correction des abus et le salut du peuple, dépendent absolument du triomphe de cette même opinion publique $»^{74}$. Bouchette répète que le public est doué de sagesse et de réflexion, et que son pouvoir et sa capacité intellectuelle contraignent ses adversaires à "respecter une population intelligente et ferme qu'hier ils osaient mépriser ${ }^{75} »$. En réponse aux propos de Parent sur les gens sans instruction, Bouchette conforte son rival: «Oui M. l'Editeur du Canadien, il est possible, il est même probable que des journaux de la forme et de la taille du Libéral, feront beaucoup de mal aux journaux de la grandeur de l'ébaissement [sic] de la trempe de celui que vous conduise ${ }^{76}$.» Parent est attaqué pour son élitisme:

$[\mathrm{P}]$ ourriez vous nous dire que sont devenus les hommes qui forment la classe ouvrière, est-ce que vous n'en voulez plus! [...] Nous sommes certains que vous auriez honte d'être soutenu par de semblables gens, vous qui êtes toujours qu'avec des honorés et favorisés par le pouvoir; c'est sans doute pour cette raison que vous avez dernièrement méprisé cette partie de vos compatriotes qui forment la classe ouvrière ${ }^{77}$.

72. L'Estafette, 6 février 1838.

73. L'Estafette, 12 janvier 1838 .

74. Le Libéral, prospectus, 17 juin 1837. Majuscules dans l'original. Voir également les éditions du 5 et du 12 septembre 1837. Cette perspective est partagée au sein du Parti patriote: La Minerve 22 juin 1837 reproduit le plaidoyer de Bouchette en faveur d’une opinion publique éclairée. C. H. O. Côté («Agricola») utilise cette même image de l'opinion publique: La Minerve, 15 mai 1837.

75. Le Libéral des 17 juin et 26 juillet 1837.

76. Le Libéral, 24 octobre 1837. Italiques dans l'original.

77. Le Libéral, 31 octobre 1837. 
Bouchette est finalement le seul éditeur à affirmer qu'il s'adresse au public «dans le but de lui faciliter les moyens de son expression». La figure de l'éditeur comme guide de l'opinion n'est pas totalement absente, mais elle s'efface derrière celle d'un éditeur qui ne fait que relayer l'opinion publique. Il répète que son journal est «un interprète impartial et fidèle» du public (patriote). Il lui a "frayé la voie pour l'expression de [ses] sentiments $[\ldots]$ le moi n'y est pour rien $»^{78}$. Sa conception n'est pas forcément contredite par son propre engagement, du moins en principe, puisque ses prises de position sont présentées comme le reflet de la volonté d'une opinion publique autonome. Bouchette et ses correspondants reprochent tout l'inverse à Parent: " vous nous avez tourné le dos et nous allons, par là, être forcés de vous le tourner à notre tour, puisque vous n'êtes plus l'écho de notre opinion ${ }^{79}$."

Si les éditeurs affirment servir les intérêts des différentes classes de la société, ce principe est relativisé par l'accessibilité à leur journal, voire par les rapports qu'ils entretiennent avec leurs employés ${ }^{80}$. Au Bas-Canada, le journal demeure un objet onéreux. L'Écho du Pays admet qu'il reste «un produit de luxe». Le montant de l'abonnement annuel est similaire pour la plupart des journaux, $4 \$$ ou 20 chelins. À ce prix, nombreux sont ceux qui ne peuvent lire le journal que par l'intermédiaire des bibliothèques, des sociétés littéraires, des cabinets de lecture et des instituts d'artisans ${ }^{81}$. Au printemps 1837, c'est presque naturellement que Le Populaire déclare se vendre au même prix. L'incohérence est cependant évidente entre vouloir parler pour «le peuple» et être vendu comme un produit de luxe. Seul Le Libéral rompt avec cette pratique. Sa partie française est vendue à moitié prix, un montant maintenu lorsque double le nombre de pages. Sous cet angle, Bouchette fait preuve de cohérence lorsqu’il déclare écrire pour un public moins fortuné.

78. Le Libéral, prospectus, 17 juin 1837. Italiques dans l'original. La représentation de l'éditeur comme guide de l'opinion est néanmoins présente dans ses propos: 12 septembre et 14 novembre 1837 . En outre, il utilise son journal et sa position d'éditeur dans la lutte politique : 15 septembre 1837.

79. Correspondance de "Qu'en pensez-vous!!», Le Libéral, 15 juillet 1837. Lire aussi la correspondance d' "Un ouvrier», Le Libéral, 3 novembre 1837.

80. Ludger Duvernay (La Minerve), Étienne Parent et Jean-Baptiste Fréchette (Le Canadien) sont, par exemple, accusés de ne pas les respecter: Robert Tremblay, «Artisans et ouvriers à l'époque des revendications démocratiques et nationalitaires du Parti patriote dans le Bas-Canada, 1832-1838: un rendezvous manqué avec l'histoire?", Bulletin d'histoire politique, 25, 2 (hiver 2017), p. 161-162.

81. J. Hamelin et A. Beaulieu, "Aperçu du journalisme...», p. 311-312; J. Hare et J.-P. Wallot, «Les entreprises... », p. 76; G. Laurence, "Les journaux... », p. 252. Pour un aperçu des salaires dans les années 1830 : R. Tremblay, «Artisans et ouvriers...», p. 150. 


\section{DISCUSSION PUBLIQUE}

Derrière ces différentes figures du public, les lecteurs ont une existence empirique. Ils s'expriment et, quoi qu'en pensent les critiques, sont naturellement doués de raison. S'il est un produit de luxe, le journal, et encore plus les propos tenus, circulent au-delà du cercle des abonnés. Les éditeurs déplorent cette pratique qu'ils sont incapables d'enrayer. L'habitude de prêter les journaux est «une fâcheuse coutume»: «Il se tire et se distribue actuellement 1000 copies de notre feuille [...] et cependant on peut compter au moins 7 ou 8000 lecteurs; donc les sept huitièmes des amateurs s'amusent aux dépens de l'autre ${ }^{82}$. Les chiffres sont similaires pour les historien(ne)s du Québec et des États-Unis, environ cinq lecteurs par exemplaire $^{83}$. À ce phénomène s'ajoute celui de la lecture publique qui rassemble des auditoires nombreux, notamment dans les campagnes. Les chiffres de vente sont donc loin de quantifier les personnes ayant accès à la discussion publique, et l'influence des journaux déborde les seuls individus fortunés et alphabétisés ${ }^{84}$.

Durant les premières décennies du XIX ${ }^{\mathrm{e}}$ siècle, le taux d'alphabétisation grimpe rapidement, notamment au Bas-Canada ${ }^{85}$. Soixante-six journaux sont créés entre 1805 et 1840 , dont plus de la moitié après $1830^{86}$. Cette augmentation découle d'une corrélation : l'expansion de la presse s'appuie sur l'élargissement du lectorat, le journal étant l’imprimé le plus accessible $^{87}$. Au sein des sociétés littéraires et des bibliothèques, les pratiques se démocratisent sous l'impulsion des artisans et des femmes. Cet élargissement de la sphère publique dans une période de tensions explique en partie la radicalisation du débat ${ }^{88}$. L'éditeur publie dans un contexte de remise en cause de l'édifice politique, social et économique, et pour convaincre, il doit aussi répondre aux attentes d'un public plus critique en période révolutionnaire ${ }^{89}$. En 1837, la discussion par voie de presse revêt

82. Le Fantasque cité dans M. Lemire, dir., La vie littéraire..., p. 175.

83. Claude Galarneau, «Livre, culture et société», Société royale du Canada. Présentation, 35 (1979-1980), p. 111 ; C. G. Steffen, «Newspapers for Free... », p. 382.

84. A. Greer, Habitants et patriotes..., p. 131.

85. Michel Verrette "L’alphabétisation», dans P. Lockart Fleming et al., dir., Histoire du livre..., p. 178-179. Allan Greer, "The Pattern of Literacy in Québec, 1745-1899», Histoire sociale/Social History, 12, 24, p. 293-335.

86. Gérard Laurence, "Les journaux dans la "Province de Québec" et au Bas-Canada», dans P. Lockhart Fleming et al. dir., Histoire du livre..., p. 250 ; J. Hare et J.-P. Wallot, «Les entreprises... », p. 78-79.

87. M. Verrette, «L’alphabétisation...», p. 175 et 179.

88. Heather Murray, "Lecteurs et société», dans P. Lockart Fleming et al., dir., Histoire du livre..., p. $186-192$.

89. M. Cambron et H.-J. Lüsenbrick, "Presse, littérature... », p. 127. Sur la position critique des lecteurs durant la Révolution française: Roger Chartier, Les origines culturelles de la Révolution française (Paris, 
même une signification particulière puisque les travaux parlementaires sont suspendus. Éditeurs et lecteurs font référence à cette dérégulation politique qui octroie aux échanges dans la presse, aux assemblées publiques et finalement à la rue un rôle politique déterminant. «[G]râce à la vacance de la législature, vous savez comme l'art d'écrire se propage dans nos campagnes!» souligne « un habitant» dans une correspondance pour Le Populaire $e^{90}$. Le caractère dialogique du journal s'en trouve renforcé ${ }^{11}$.

Le traitement des correspondances des lecteurs et lectrices est un indicateur notable de la démarche des éditeurs vis-à-vis du public. La pratique épistolaire s'inscrit dans ce contexte de prise de parole et de démocratisation de la réflexion publique. Écrire au journal signifie «entrer dans la sphère de discussion publique, participer à un débat d'idées et par là-même affirmer sa légitimité de citoyen ${ }^{92}$.» En pratique cependant, la fabrication des voix des lecteurs est un trait général des journaux au XIX ${ }^{\mathrm{e}}$ siècle, surtout dans la presse littéraire et culturelle ${ }^{93}$. Sous réserve de leur authenticité, Gérard Laurence remarque en outre que les correspondances des lecteurs sont, au Bas-Canada, tamisées et orientées, ce qui signifie que la discussion est davantage un forum de partisans plutôt qu'une agora où s'expriment des idées contraires ${ }^{94}$. Ces correspondances font néanmoins du journal un espace d'échanges et de médiation dans lequel les lecteurs sont invités à dialoguer ${ }^{95}$. La prise en compte sincère de leur voix est une démarche éditoriale éminemment politique, et absolument démocratique $^{96}$. Cette pratique permet aussi au public de jouer un rôle important:

Le Seuil, 1990); Béatrice Didier, Écrire la Révolution, 1789-1799 (Paris, Presses universitaires de France, 1999).

90. Le Populaire, 10 juillet 1837.

91. Pour une réflexion sur l'interaction des lecteurs avec les journaux, lire E. Absalyamova et V. Stiénon, dir., Les voix du lecteur..., et Guillaume Pinson, dir., La lettre et la presse: poétique de l'intime et culture médiatique, <www.medias19.org/index.php?id=275>.

92. Éric Wauters, «Le procédé épistolaire dans la presse française de la Révolution à la Restauration ", dans G. Pinson, dir., La lettre et la presse... < http://www.medias19.org/docannexe/file/327/ wauters. pdf $>$.

93. Valérie Stiénon, «Lecteurs truqués: sur la fabrique médiatique du lectorat au XIX ${ }^{e}$ siècle», dans E. Absalyamova et V. Stiénon, dir., Les voix du lecteur..., p. 189.

94. G. Laurence, «Les journaux...», p. 251.

95. Alain Vaillant, «Le double jeu du journal, entre communication médiatique et correspondance privée», dans G. Pinson, dir., La lettre et la presse... : <http://www.medias 19.org/index.php?id=341>; M. Bédard, Écrire en temps d'insurrections..., p. 45.

96. Elina Absalyamova, "Notre téléphone”, une rubrique de La Nouvelle Rive Gauche/Lutèce en tant que chambre d'écho", dans E. Absalyamova et V. Stiénon, dir., Les voix du lecteur..., p. 205-219. 
le lecteur, devenu correspondant, devient une précieuse source d'informations ${ }^{97}$.

Ces correspondances posent donc «la question des rapports du journaliste à son public, à la pratique de son métier et à son rôle dans la citée8 ${ }^{98}$. Le cas bas-canadien fait néanmoins écho aux études portant sur la France ${ }^{99}$. Marconnay, par exemple, présente son journal comme un lieu de discussion ouvert «à toutes les opinions», y compris celles «qui pourraient contrarier nos vues", mais il prévient aussi qu'elles doivent respecter certaines règles de convenance, comme celle de «ne point allumer les passions ${ }^{100}$. En plus de décider de leur pertinence, et in fine de leur publication, il se réserve aussi le droit de les commenter. Comme annoncé, il publie rapidement de nombreux textes de ses lectrices. Observer cette démarche chez un adversaire du Parti patriote, et inversement la quasiabsence des plumes féminines dans La Minerve et Le Libéral, semblent confirmer la posture misogyne des révolutionnaires ${ }^{101}$. La discussion contradictoire est par contre minimale dans les colonnes du Populaire. L'écrasante majorité des lettres publiées sont des critiques des républicains, dont les voix sont absentes. Le public participe à une discussion collective, du moins figurée, mais celle-ci se fait par journaux interposés, les correspondants du Populaire répondant aux propos tenus dans les feuilles radicales ${ }^{102}$. Rares sont les exceptions, comme celle où «François » accuse Marconnay de manquer d'honneur, attaque à laquelle il répond sèchement "en l'assurant de notre profond mépris » ${ }^{103}$ - le caractère insultant de la correspondance laisse ici supposer de son authenticité. Lorsqu'elle est jugée convenable, c'est-à-dire dénuée de propos sympathiques au Parti patriote, la discussion sereine prend forme, soit avec l'éditeur, soit avec les lecteurs, d'une manière très similaire aux pratiques de L'Abeille de la Nouvelle-Orléans ${ }^{104}$. Pour son opposant de L'Estafette, ces

97. Fernande Roy et Jean de Bonville, «La recherche sur l’histoire de la presse québécoise. Bilan et perspectives", Recherches sociographiques, 41, 1 (2000), p. 22.

98. E. Wauters, «Le procédé épistolaire...».

99. E. Absalyamova et V. Stiénon, dir., Les voix du lecteur...; G. Pinson, dir., La lettre et la presse...

100. Le Populaire, 10 avril 1837.

101. Allan Greer, «La république des hommes: les patriotes de 1837 face aux femmes», Revue d’histoire de l'Amérique française, 44, 4 (1991), p. 507-528.

102. Cette discussion par journaux interposés est par exemple observable dans Le Populaire des 4, 16, 21 et 28 août 1837.

103. Le Populaire, 26 juillet 1837.

104. Par exemple, une discussion sur un ouvrage de mathématique occupe les lecteurs durant le mois de juillet 1837. Pour un échange avec Marconnay, Le Populaire, 28 juillet 1837. Voir notamment L'Abeille du 19 janvier 1838, lorsque les lecteurs entament une discussion à propos des loteries. 
écrits semblent authentiques, ces correspondants étant mobilisés pour peser dans la joute verbale ${ }^{105}$.

Étienne Parent insère beaucoup moins de correspondances ${ }^{106}$. Il est probable qu'il en reçoive ou en sollicite moins, mais nous remarquons aussi l'absence d'une représentation du public comme acteur de la discussion, ce qui concorde avec sa pensée selon laquelle l'éditeur guide l'opinion. Il refuse de publier des correspondances critiques envers les autorités, ce qui semble signifier que ces correspondances refoulées sont authentiques, et il s'en explique: l'une d'elles est ainsi rédigée par "une tête qui a besoin d'être refroidie ${ }^{107} »$. Dans son rapport au lecteur, Parent fait donc écho à l'idéal philanthropique de la connaissance établissant les élites comme les seuls producteurs raisonnables d'opinions ${ }^{108}$.

La discussion contradictoire n'est pas davantage présente dans le Libéral, mais l'espace occupé par les lecteurs est bien plus conséquent. Un public souvent anonyme - et probablement fictif quant à la signature - argumente contre les hommes de lettres, du moins ceux qui s'opposent au Parti patriote. Un «Indigné» déclare que les mensonges de Parent «m’obligent, moi, pauvre campagnard illettré, à prendre la plume pour les contredire ${ }^{109}$ ». Avec ce campagnard illettré prenant la plume pour s'exprimer, serions-nous là dans l'illustration la plus parfaite d'un public figuré? Un électeur de Portneuf affirme, pour sa part, que des "pauvres cultivateurs nullement initiés aux difficultés de la politique» possèdent la capacité, morale et intellectuelle, de répliquer aux élites ${ }^{110}$. Authentique ou non, c'est le signifiant qui importe ici: en ces temps révolutionnaires, l'élite ne peut plus revendiquer le monopole de la raison. En réponse à un article de L'Ami du Peuple, Le Libéral publie une lettre présentée aux lecteurs comme étant celle de paroissiens de Sainte-Anne-de-Lapérade, ces derniers s'interrogeant sur les «qualités intellectuelles » du «cerveau malade » qui dirige ce journal. L'audace de ces lettres, même fictives, est d'affirmer que des habitants remettent publiquement en cause l'intelligence d'un

105. L'Estafette, 6 mars 1838.

106. Sur un échantillon (le mois d'août 1837), Le Canadien publie en moyenne 1,25 correspondance par numéro, contre 2,3 pour Le Populaire et 3,25 pour Le Libéral. Autre indication, Le Libéral du 2 août est majoritairement composé de lettres de lecteurs.

107. Le Canadien, 2 octobre 1837. Cette correspondance est finalement publiée dans Le Libéral du 17 juin 1837.

108. Dans cette veine, une intéressante étude de cas est réalisée par Anaïs Goudmand, «La voix des ouvriers: Les Mystères de Paris, espace de dialogue entre Eugène Sue et ses lecteurs?», dans E. Absalyamova et V. Stiénon, dir., Les voix du lecteur..., p. 59-72.

109. Le Libéral, 18 août 1837.

110. Le Libéral, 3 octobre 1837. 
homme de lettres ${ }^{111}$. Parallèlement, la mise en forme (ou en scène) exprime un message limpide: Le Libéral est la voix d'un public actif qui possède la légitimité intellectuelle de critiquer les hiérarchies. Bouchette publie en novembre 1837 une missive d' "Un ouvrier», une réponse aux propos de Parent sur les gens sans instruction. Cette lettre rappelle que Parent ne doit son succès qu'au peuple, qu'il méprise désormais. Pour l'ouvrier, le citoyen est celui qui lit et s'informe. Or, Parent refuse ce statut à «la classe pauvre et ouvrière», alors même que, «bien que son papier soit de quatre piastres par année», le lectorat ouvrier constitue "une grande partie de ses abonnés». Il est donc probable que l'ouvrier ne le soit que de pseudonyme, ou alors que le terme englobe des artisans pouvant s'offrir un journal, mais c'est le même message révolutionnaire qui ressort: grâce à son accès à la discussion publique, le menu peuple possède le droit et la capacité de contredire le dénigrement des hommes de lettres, d'affirmer son pouvoir et d'écrire l'histoire. Le public rébellionnaire participe, au moins dans sa représentation, à l'ébranlement de l'ordre établi jusque dans les rapports au savoir: «Quelle effronterie de nous faire croire que les riches ont seuls le droit de dicter les volontés du peuple et d'être appelés enfan[t]s de la patrie $»^{112}$.

Les éditeurs affirment tous détenir la vérité et accusent leurs adversaires d'être dans l'erreur, mais pour ce faire il leur faut convaincre les lecteurs et ne pas seulement déclamer cette vérité. Les dires et les preuves avancées sont épiés, critiqués et contredits. La démonstration de la vérité passe par le contre-argumentaire permanent. Les controverses sont nombreuses, souvent longues et documentées. Elles peuvent porter sur des aspects très secondaires en ces temps révolutionnaires. L'une d'elles concerne la réunion des patriotes du Saguenay du 25 juin 1837: jusqu'en novembre, les journaux reviennent sur cet événement pour disserter sur les réalités de l'opinion publique ${ }^{113}$. Dans ces échanges, les preuves utilisées sont régulièrement les témoignages du public. La pratique épistolaire n’est donc pas une simple conversation, mais une coopération éditoriale ; le lecteur participe à l'écriture du journal, et par ce biais à la discussion publique. L'éditeur s'efface même parfois, comme Bouchette qui reconnaît ne pas posséder une connaissance suffisante pour soutenir un débat théologique

111. Le Libéral, 25 août 1837.

112. “Un ouvrier» dans Le Libéral, 3 novembre 1838.

113. Le Libéral, 8 juillet 1837. Lire par exemple comment Charles Drolet, élu du comté de Saguenay, cherche à "prouver» la véracité du rapport de Bouchette: Le Libéral, 29 juillet 1837. 
avec L'Ami du Peuple, sollicitant un nommé «Contrebandier» pour répliquer à son confrère ${ }^{114}$.

À l'écart des tensions du contexte bas-canadien, Robinson met davantage en pratique la discussion contradictoire. Dès les premières semaines, nous observons un échange trans-partisan et transfrontalier avec ses adversaires du Bas-Canada sur la légalité de la loi martiale ${ }^{115}$. Cette discussion s'ajoute au débat permanent avec L'Ami du Peuple, "une discussion que nous ne croyons pas sans utilité», et dont le "public seul est juge »"16. Le public ne prend pas la parole dans ces échanges, mais les éditeurs loyalistes comme radicaux s'accordent pour tenter de le convaincre par l'argument et la preuve - le lectorat ayant accès aux idées adverses.

En mars 1838, Robinson se lance dans une discussion avec un Montréalais anonyme. Ce lecteur mécontent l'accuse de pousser les Canadiens vers un massacre certain, car il s'est «laissé séduire par le terme talismanique de liberté; malheureuse abstraction dont on a trop abusé». Ce correspondant souhaite que les éditeurs usent de leur pouvoir pour guider la réflexion des lecteurs, «enseigner aux peuples plutôt que de les provoquer au soulèvement et au carnage ». Robinson répond en explicitant son engagement: «La liberté, cette fille aînée de l'intelligence, a produit plus de bien réel et fait verser moins de sang [...] que le système monarchique». Il légitime donc les révolutionnaires en les associant explicitement à la notion d'intelligence. Robinson accepte de publier le contreargumentaire du Montréalais, qui témoigne du dynamisme et de la complexité de la réflexion publique puisqu'il précise qu'il n'est pas opposé à la liberté et voit la "démocratie» comme la forme de contrat social la plus propre à la diffuser. Ce qu'il réclame est davantage de réflexion: «Ce contre quoi je m'élève et proteste, c'est la vague signification qu'on a jusqu'à ce jour attribuée à ce terme complexe. » Le correspondant expose ensuite une cosmologie singulière dans laquelle la liberté est définie comme la possibilité de vivre sans peur, privilège unique des animaux sauvages. Ainsi, «ni vous, ni moi, ni aucun individu que ce soit, même parmi les plus riches, et, à plus forte raison, dans l'immense masse des pauvres, ne jouit de la liberté». L'éditeur new-yorkais met fin à la discussion en recommandant la lecture de La Fontaine plutôt que Locke et Montesquieu ${ }^{117}$.

114. Le Libéral, 12 septembre 1837.

115. L'Estafette, 16 février 1838.

116. L'Estafette, 6 mars 1838.

117. L'Estafette des 16 mars et 3 avril 1838. 
Les autorités britanniques bloquent matériellement ce type d'échanges au printemps 1838, interdisant aux journaux étasuniens pro-patriotes l'accès à la colonie ${ }^{118}$. Contrairement à Parent par exemple, Robinson, et à un degré moindre Le Populaire et L'Ami du Peuple, conçoivent et appliquent la discussion publique de façon plus démocratique. Conscients du sens de leurs échanges, ces éditeurs ont inévitablement œuvré pour susciter la réflexion en accueillant les contre-argumentaires dans leurs colonnes. Cette réalité suggère l'existence d'une relation éditeur-public plus complexe que celle d'un simple guide vers un lectorat passif, et semble confirmer l'hypothèse d'une certaine autonomie intellectuelle du public que les hommes de lettres ne pouvaient ignorer - à quoi bon se lancer dans un périlleux débat public s'il suffisait d'affirmer une vérité? Le lecteur doit être convaincu, et pas seulement instruit ou informé.

\section{CONCLUSION}

En analysant le journal comme espace d'interactions, nous constatons que le public est omniprésent dans la plupart des journaux francophones, soit à travers sa voix réelle ou fabriquée, soit en contraignant les éditeurs à repenser leur rapport pratique et conceptuel au public. Si la mise en pages d'une discussion citoyenne demeure avant tout une représentation $^{119}$, les éditeurs francophones nord-américains durant la Rébellion ont l'obligation de s'adresser à l'intelligence du public, sous peine de perdre rapidement toute influence, ce qui arrive au Canadien et au Courrier des États-Unis. Ce phénomène est accentué par la crise révolutionnaire dont l'enjeu est, précisément, le pouvoir populaire. En utilisant le journal comme miroir, il apparaît que de nombreux lecteurs se laissent convaincre par la démonstration plus que par l'éloquence ou la réputation. La presse républicaine fait globalement preuve d'un esprit démocratique plus marqué dans ses rapports pratiques et théoriques avec le public. Cet engagement s'observe lorsque sa représentation est invoquée, lorsque les colonnes sont davantage ouvertes aux correspondances, ou lorsque la discussion contradictoire est publiée. Inversement, plusieurs éditeurs loyalistes se présentent comme des guides intellectuels. Mais il ne s'agit pas d'un déterminisme strict; l'éditeur, quel que soit son positionnement politique, est l'arbitre de la démocratisation de son journal. Les journaux républicains bas-canadiens sont tout aussi réticents à imprimer des contre-

118. Voir le constat de L'Estafette à ce propos (8 mai 1838).

119. E. Absalyamova et V. Stiénon, dir., Les voix du lecteur... 
argumentaires, et certains éditeurs loyalistes œuvrent également pour démocratiser la discussion publique. Au-delà de ces pratiques éditoriales, les indices concordent aussi pour soutenir l'hypothèse que le public francophone possède, dans sa diversité, une relative autonomie intellectuelle face à la plume des éditeurs, au point de proposer certaines cosmologies singulières dans l'arène du débat public. Pour pouvoir lire et entendre ce public, il semble cependant primordial de le concevoir dans sa pluralité, d'explorer les opinions des publics, mais aussi les échanges variés entre les membres du public - plus qu'une représentation vague de l'opinion publique qui semble résulter avant tout de la rhétorique politique ou philosophique $^{120}$.

Jürgen Habermas a accepté de considérer cette pluralité qui relativise l'hégémonie de la bourgeoisie dans l'espace public ${ }^{121}$. Dans son étude initiale de l'opinion publique glorifiant les Lumières, la sphère publique plébéienne, à laquelle semble appartenir le «Vieux de la Montagne» par exemple, était en effet absente de l'histoire. Ces oubliés constituent pourtant un public actif ${ }^{22}$. Arlette Farge, dans son étude de l'opinion plébéienne parisienne au XVIII ${ }^{\mathrm{e}}$ siècle, soutient que tout individu a compétence à la critique. Certaines recherches sur le Bas-Canada sont convergentes puisqu'elles démontrent que les habitants des campagnes comme les artisans ne suivaient pas aveuglément les dirigeants du Parti patriote ${ }^{123}$. Reconnaître cette capacité intellectuelle du public, ce qui ressort du présent article, doit nous permettre de prendre nos distances avec les thèses insistant sur la manipulation des opinions par les grands noms de la Rébellion $^{124}$. Identifier l'existence de plusieurs sphères n'est qu'une étape vers une nouvelle conception du public, duquel ne doivent plus être exclus un milieu social, les femmes, ou un groupe culturel. Il deviendrait alors possible d'évaluer plus en détail les cheminements des idées, leur fermen-

120. G. Pinson, La culture médiatique..., p. 59.

121. Jürgen Habermas, L'espace public. Archéologie de la publicité comme dimension constitutive de la société bourgeoise (Paris, Payot, 1993 [1962]), p. v-vi. La préface de 1990 dans laquelle il reconnaît cette erreur a été publiée en français dans Quaderni, 18 (1992), p. 164.

122. Le premier ouvrage majeur en ce sens est celui d'Edward P. Thompson, La formation de la classe ouvrière anglaise (Paris, Seuil, 1988 [1963]). Une autre étude majeure est celle de Roger Chartier, Origines culturelles de la Révolution...

123. Arlette Farge, Dire et mal dire. L'opinion publique au XVIII siècle (Paris, Seuil, 1992), p. 17. Mona Ozouf confirme les conclusions d'Arlette Farge: "Quelques remarques sur la notion d'opinion publique au XVIII siècle», Réseaux, 5, 22 (1987), p. 85-86. Pour le Bas-Canada: A. Greer, Habitants et Patriotes...; R. Tremblay, "Artisans et ouvriers...».

124. Thèse notamment défendue par Fernand Ouellet, Éléments d'histoire sociale du Bas-Canada (Montréal, Hurtubise HMH, 1972). 
tation et leurs altérations, et donc les mécanismes de l'opinion publique ${ }^{125}$. De nombreux historien(ne)s souhaitent désormais rompre avec l'élitisme qui caractérise la schématisation d'Habermas ${ }^{126}$. Ce chemin nous conduira vers un public qui, malgré tous ses défauts et incohérences, ne sera plus assimilé à une foule dénuée de subtilité, qui étonnera peut-être par sa raison, ses opinions et ses innovations, s'apparentant davantage au public décrit par Gabriel Tarde et John Dewey ${ }^{127}$.

125. L'intérêt heuristique d'une telle démarche a été maintes fois souligné : Geoff Eley, «Nations, Publics, and Political Cultures: Placing Habermas in the Nineteenth-Century", dans Craig J. Calhoun, dir., Habermas and the Public Sphere (Cambridge, The MIT Press, 1992), p. 289-339; Mary Ryan, "Gender and Public Access: Women's Politics in Nineteenth-Century America ", dans C. J. Calhoun, dir., Habermas..., p. 259-288; Michael Hoffman, Habermas's Public Sphere: A Critic (Lanham, Fairleigh Dickinson University Press, 2017), p. 106-126; Fabrice Erre, «Le "Roi-Jésuite” et le "Roi-Poire” : la prolifération des "espiègleries" séditieuses contre Charles X et Louis-Philippe (1826-1835)", Romantisme, 150, 4 (2010), p. 113 ; Nelleke Moser, «Manuscript Pamphlets and Made-Up Performances: New Sources and Challenges in the Study of Public Opinion ", dans Jan Bloemendal, Arjan van Dixhoorn et Elsa Strietman, dir., Literary Cultures and Public Opinion in the Low Countries, 1450-1650 (Leiden, Brill, 2011), p. 181-218; Filippo De Vivo, "Public Sphere or Communication Triangle? Information and Politics in Early Modern Europe», dans Massimo Rospocher, dir., Beyond Public Sphere. Opinions, Publics, Spaces in Early Modern Europe (Bologne/ Berlin, Il Mulino/Duncker \& Humblot, 2012), p. 115-136; Alexandre Neumann, «L'espace public oppositionnel: lorsque l'oikos danse dans l'agora », Cahiers Sens public, 15-16, 1 (2013), p. 57-58.

126. Voir en particulier M. Rospocher, dir., Beyond Public Sphere... Un récent ouvrage sur l'opinion publique aux États-Unis relègue la discussion sur Habermas dans son épilogue: Mark G. Schmeller, Invisible Sovereign: Imagining Public Opinion from the Revolution to Reconstruction (Baltimore, Johns Hopkins University Press, 2016), p. 224-225. Pour une rapide vue d'ensemble des critiques adressées à Habermas: Harold Mah, «Phantasies of the Public Sphere : Rethinking the Habermas of Historians », The Journal of Modern History, 72 (mars 2000), p. 153-182.

127. Gabriel Tarde, L'opinion et la foule, (Paris, PUF, 1989 [1901]); John Dewey, Le public et ses problèmes, traduction de Joëlle Zask (Paris, Gallimard, 2010 [1927]). 